\title{
Millimeter-Wave MIMO Balanced Antipodal Vivaldi Antenna Design for Autonomous Cars
}

\author{
Ali Araghi ${ }^{1}$, Mohsen Khalily ${ }^{2}$, Pei Xiao ${ }^{2}$, Arash Kosari ${ }^{1}$, Houman Zarrabi ${ }^{1}$, Rahim Tafazolli ${ }^{2}$ \\ ${ }^{1}$ Communication Technology Group, Iran Telecommunication Research Center (ITRC), Tehran, Iran, \\ \{a.araghi, a.kosari, h.zarrabi\}@itrc.ac.ir \\ ${ }^{2}$ Institute for Communication Center, Home of 5G Innovation Center, University of Surrey, Guildford, UK, GU2 7XH, \\ \{m.khalily, p.xiao, r.tafazolli\}@surrey.ac.uk
}

\begin{abstract}
This paper introduces a millimeter-wave multipleinput-multiple-output (MIMO) antenna for autonomous (selfdriving) cars. The antenna is a modified four-port balanced antipodal Vivaldi which produces four directional beams and provides pattern diversity to cover 90 deg angle of view. By using four antennas of this kind on four corners of the car's bumper, it is possible to have a full $360 \mathrm{deg}$ view around the car. The designed antenna is simulated by two commercially full-wave packages and the results indicate that the proposed method can successfully bring the required $90 \mathrm{deg}$ angle of view.
\end{abstract}

Keywords-multiple-input-multiple-output (MIMO); millimeter wave; balanced antipodal Vivaldi antenna (BAVA); pattern diversity.

\section{INTRODUCTION}

Self-driving, autonomous cars have long been a stimulating idea for both industry and academia. A number of studies have been conducted in this regard which brings this idea close to reality, to the extent that companies like Tesla, Delphi, and Google are trying to manufacture fully autonomous cars [1]. An autonomous car generally relies on three complementary technologies to sense its surroundings: an array of video camera, a LIDAR system, and a maximum 250m-range Radar system [2]. The cameras enable the car to have a perspective view of the area while the LIDAR system provides a primary vision of obstacles around the car. Detailed-view of the environment near the car is mainly achieved by the Radar system operates in the $76-77 \mathrm{GHz}$ band with $1 \mathrm{GHz}$ bandwidth [3]. Recently, millimeter-wave bands have attracted much interest by the research community [4], [5] since it will provide lots of advantages ${ }^{1}$. To have an appropriate operation of autonomous cars, it is important that the three aforementioned technologies work together simultaneously and consistently, but it can be claimed that the safety of drive is mainly tied in with its Radar performance since the car's awareness of its close obstacles and blockages will be achieved by this system. Apart from that, Radar's functionality is directly affected by its antenna. Therefore, antenna design for self-driving cars will be

1 One of the major advantages offered by the millimeter-wave frequency bands is the possibility of achieving multi-gigabit wireless communications (chip-to-chip, vehicle to vehicle (V2V), and vehicle to infrastructure (V2I) communications) and ultra-high resolution automotive radars (short range radar (SRR) and long range radar (LRR)) for adaptive cruise control (ACC) and for Lane Assistance and Collision Avoidance Systems in smart vehicles. of utmost importance when autonomous cars are produced on industrial scale.

In the literature, a great proportion of studies is focused on Radar system design and its performance enhancement [2], [6], [7], or the overall Radar behavior in different cases based on test measurements [7], [8]. The status of antenna design development for autonomous cars is far from comprehensiveness and is subject to much improvement based on further breakthrough and innovations.

The main purpose of this work is to propose a novel design for a mm-wave antenna to be employed in self-driving cars. The antenna should be sensitive to the direction of arrival. This requirement is met in our design by utilizing pattern diversity in a multiple-input-multiple-output (MIMO) concept [9]. The details about antenna requirements and the proposed design are discussed in Section II and III respectively, whilst in Section $\mathrm{IV}$, the simulation results are presented. Finally, the conclusions of this study are drawn in Section V.

\section{ANTENNA REQUiREMENTS AND Placement SCENARIO}

\section{A. Antenna Requirements for Self-driving Cars}

The first consideration regarding the autonomous car's antenna, is the ability to have $360 \mathrm{deg}$ of view. Technically, the radiation pattern should be in a way that the antenna radiates in different directions to cover all $360 \mathrm{deg}$ around the car. Under this circumstance, the antenna will help the Radar system to understand the direction of arrival.

Since the operating frequency band is $76-77 \mathrm{GHz}$, the path loss will be significantly high which should be compensated by the antenna performance. Therefore the antenna gain is preferred to be medium to high.

Finally, it is worth mentioning that although the antenna will be mounted on a car's body and the weight and even the size of antenna will not be a noticeable issue from a car's point of view, it is always preferred to have a low-weight, lowprofile, and easily installable antenna with respect to the structural physics of the car for self-driving applications.

\section{B. Antenna Placement Proposed Scenario}

Antenna design for autonomous cars starts from planning on the position where the antenna is going to be mounted on 


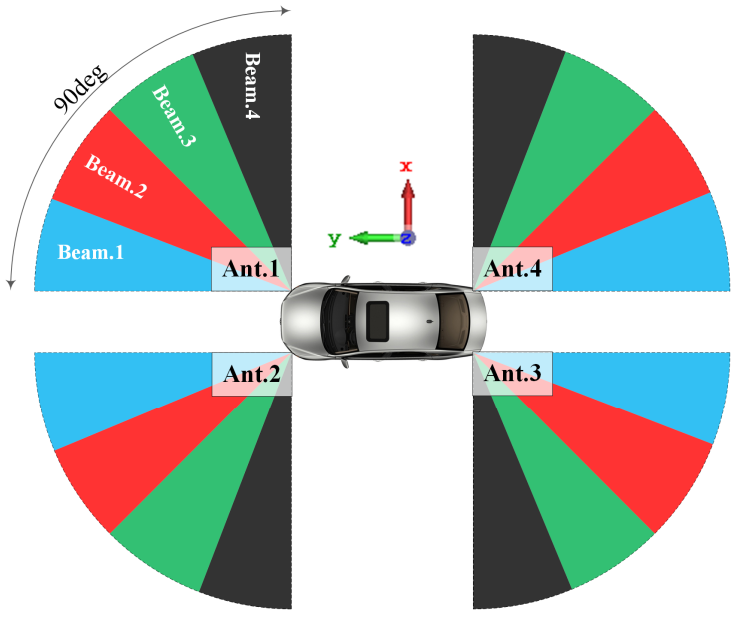

Fig. 1. The proposed antenna placement scenario. Each antenna has four ports and four corresponding directional beams, resulting in four MIMO antennas on four corners of the car.

the car and how its radiation pattern should be developed. Second task is to select a proper antenna to achieve the desired radiation pattern in the $76-77 \mathrm{GHz}$ band.

Regarding the first pointed task, Fig. 1 presents the proposed scenario which to some extent is similar to that of presents in [2]. As it is clear from this figure, four antennas are placed on four corners of car's bumper. Each antenna provides a 90 deg angle view which results in a full 360 deg view in case of four. To enable the car to detect the direction of possibly existing obstacles, each $90 \mathrm{deg}$ angle of view is divided into four, and therefore, the antenna is preferred to benefit from four directional beams. The antenna should have a diversified radiation pattern at each $90 \mathrm{deg}$ slice. If for each radiation pattern, a dedicated port is set, the antenna could act as a MIMO antenna with pattern diversity. In summary, the aim is to design a four-port MIMO antenna with four directional radiation pattern to cover a $90 \mathrm{deg}$ angle of view. Using four antennas of this kind on four corners of the car will provide a full 360 deg view around the car.

Bearing in mind the requirements and placement issue, the antenna design is presented in the following section.

\section{MIMO BALANCED ANTIPODAL VIVALDi ANTENNA DESIGN FOR SELF-DRIVING CARS}

Vivaldi antennas [10] have attracted considerable attentions since they offer high flexibility to be adjusted for a number of applications. Balanced antipodal Vivaldi antennas (BAVAs) [11] is a modified type of antipodal Vivaldi antenna that is embodied by striplines.

Integration with striplines have an eye-catching benefit for our design, the reason for this claim is the fact that antenna will going to be placed close to the outer boundaries of a car with reflective metallic surface. As a result, the antenna performance could be affected by the car's body. Apart from that, the antenna will incorporate four ports and for each port, there must be a transmission line trace. In case of stripline, the signal trace will be sandwiched by two ground plates, mitigating the effect of environmental interference and the car on the signal trace. Moreover, antipodal Vivaldi antennas could (a)
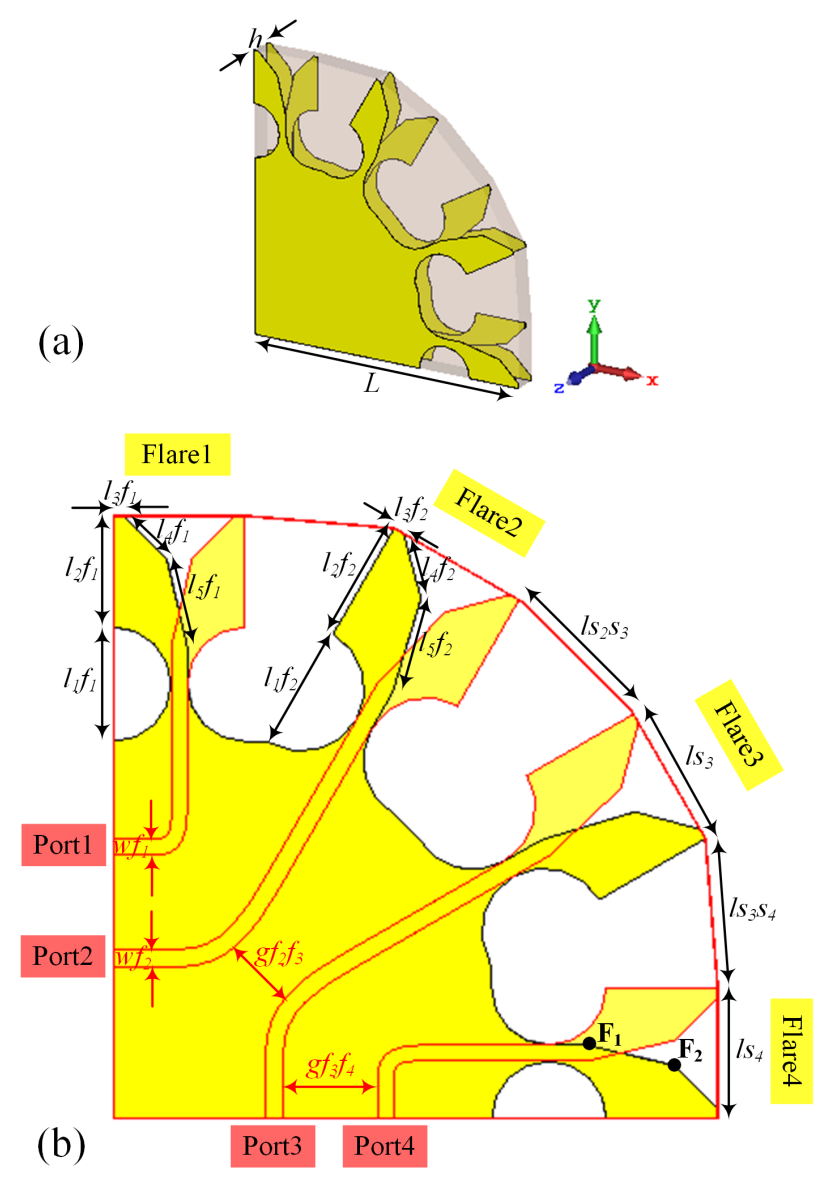

Fig. 2. The Antenna structure. (a) Perspective view, (b) view from the top. The red boundaries of conducting plate specify the middle placed layout.

TABLE I. OPTIMIZED DiMENSSIONS OF THE PROPOSED MIMO ANTENNA

\begin{tabular}{|c|c|c|c|}
\hline Parameter & $\begin{array}{c}\text { Dimension } \\
(\mathrm{mm})\end{array}$ & Parameter & $\begin{array}{c}\text { Dimension } \\
(\mathrm{mm})\end{array}$ \\
\hline$L$ & 9.51 & $l_{4} f_{2}$ & 1.07 \\
\hline$h$ & 0.776 & $l_{5} f_{2}$ & 1.55 \\
\hline$l_{1} f_{1}$ & 1.80 & $w f_{1}$ & 0.25 \\
\hline$l_{2} f_{1}$ & 1.76 & $w f_{2}$ & 0.28 \\
\hline$l_{3} f_{1}$ & 0.15 & $g f_{2} f_{3}$ & 1.21 \\
\hline$l_{4} f_{1}$ & 0.97 & $g f_{3} f_{4}$ & 1.49 \\
\hline$l_{5} f_{1}$ & 1.41 & $l s_{2} s_{3}$ & 2.45 \\
\hline$l_{1} f_{2}$ & 1.98 & $l s_{3}$ & 2.32 \\
\hline$l_{2} f_{2}$ & 1.93 & $l s_{3} s_{4}$ & 2.32 \\
\hline$l_{3} f_{2}$ & 0.16 & $l s_{4}$ & 2.11 \\
\hline
\end{tabular}

have medium to high end-fire gain in mm-wave band [12], [13].

The proposed 4-port MIMO antenna is presented in Fig. 2 and Table I provides the details of the optimized dimensions of the antenna. Commercially available FerroA6 LTCC is used as 
the substrate. The reported relative permittivity and loss tangent are 5.7 and 0.002 (at $10 \mathrm{GHz}$ ) respectively and a thickness of $0.388 \mathrm{~mm}$ is chosen. The conductor layers are silver with $97 \mu \mathrm{m}$ thickness.

BAVAs are famous for their ultra-wideband performance which is achieved when the antenna flares are tapered exponentially as reported in [14]. However, for autonomous car applications, the bandwidth is limited to $1 \mathrm{GHz}$ as discussed in the previous section. Therefore, the exponential tapering of flares is not recommended. Instead, the flares are linearly tapered with two fraction points $\mathbf{F}_{1}$ and $\mathbf{F}_{2}$. The location of these points could be optimized to adjust the operating frequency to the desired band.

The design began with optimizing the dimensions of one flare, e.g. flare 1, and then placing the other flares near it. It is important that the placement of other flares results in the minimum possible degradation of flare 1's performance. The uniform placement and rotation of flares $2 \sim 4$ shifted the operating frequency and deteriorated the direction of radiation pattern. To have a control on the operating frequency, flares 2 and 3 become a slightly larger in size compared to flares 1 and 4. The other option to optimize the operating frequency is to change the fraction points $\left(\mathbf{F}_{1}\right.$ and $\left.\mathbf{F}_{2}\right)$ of flares 2 and 3 in comparison with that of flares 1 and 4 . However, our
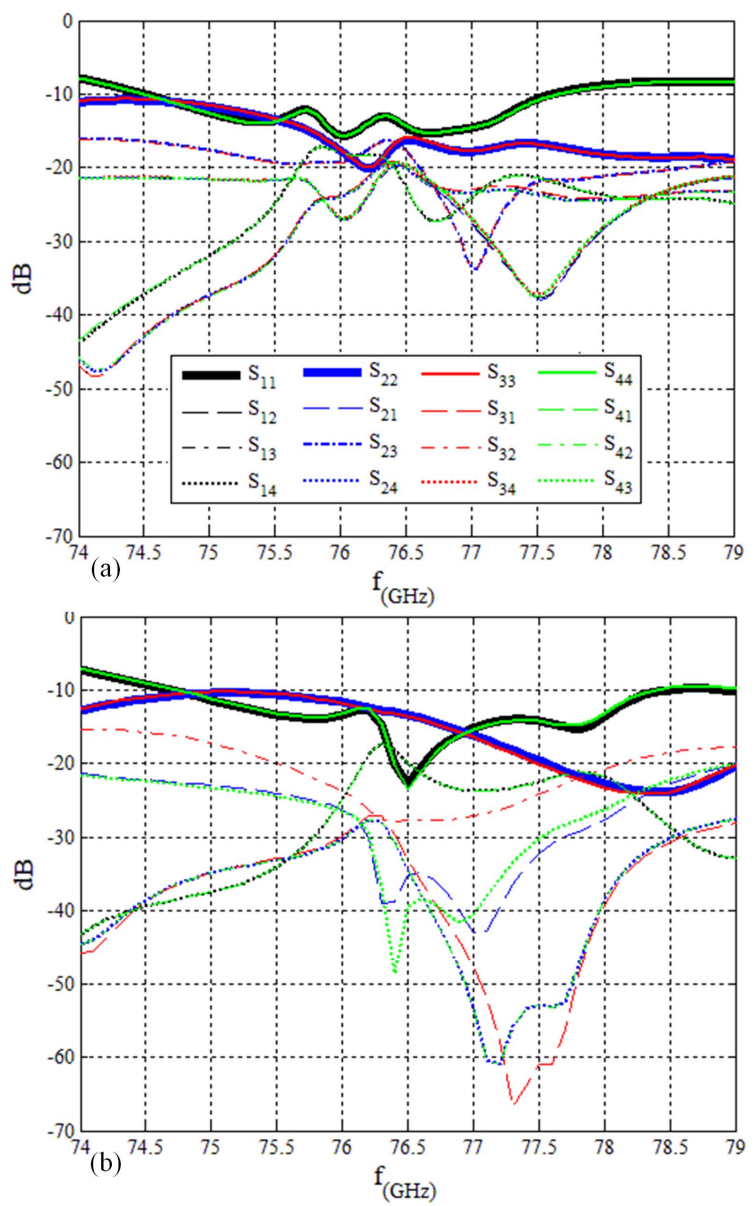

Fig. 3. Scattering parameters of the proposed antenna. (a) CST results and (b) HFSS results.

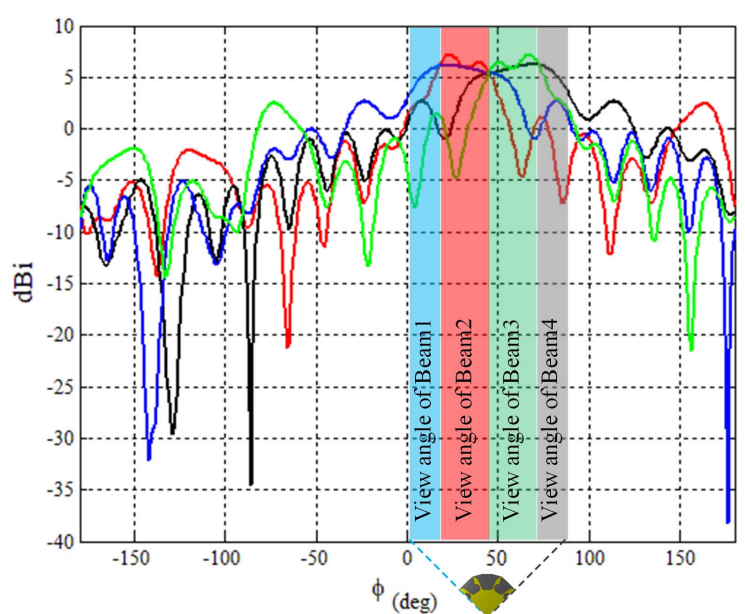

Fig. 4. Radiation pattern in xy-plane. Pattern diversity is provided by the proposed antenna.

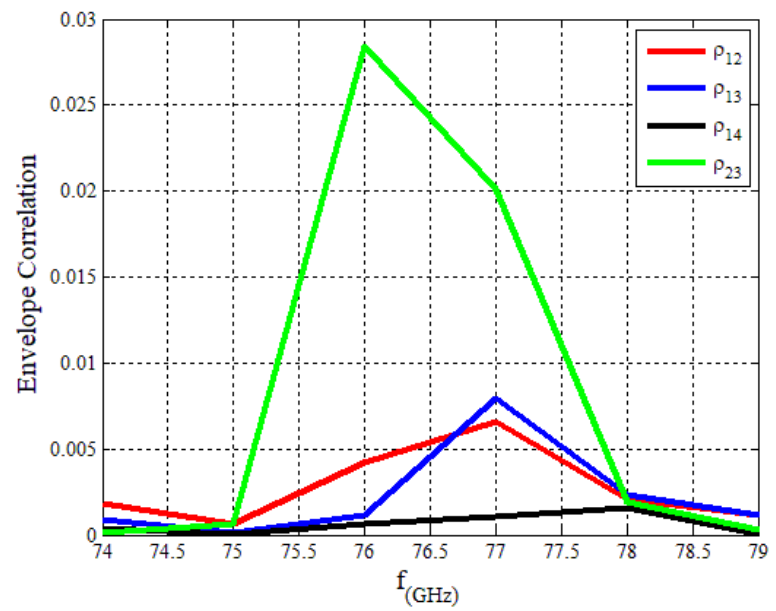

Fig. 5. Two-by-two envelope correlation coefficient of the proposed MIMO antenna.

conducted studies have shown that size optimization (first approach) is the more efficient option for the discussed antenna. Furthermore, flares 3 and 4 are set to be mirrors of flares 1 and 2; this will help the antenna radiation pattern to be restricted to the desired angle of view. The gap between nearby transmission lines $\left(g f_{n} f_{n+l}\right)$ was optimized to minimize the scattering parameter between the ports to yield a MIMO performance. It is notable that the transmission lines must be bended smoothly since sharp angles could lead to disturbances on the operating frequency.

\section{RESULTS AND DISCUSSIONS}

The proposed MIMO antenna is designed and simulated by two commercial full-wave packages, CST Microwave Studio and Ansys HFSS. Fig. 3 provides a complete behavior of the antenna's $S$-parameter. As depicted in this figure, the antenna benefits from a suitable $S_{n n}$ behavior in its all four ports. Simultaneously, $S_{n m}$ is kept less than $-15 \mathrm{~dB}$ in the wanted band thanks to the parametric studies discussed in the previous section. The radiation pattern of ports $1 \sim 4$ in $x y$-plane $(\theta=90$ deg) with respect to the axis presented in Fig. 1 and Fig. 2 is 
illustrated in Fig. 4 which properly meet the defined scenario presented in Fig. 1 through a 90 deg view. Beams 1 and 4 have $6.2 \mathrm{dBi}$ gain with radiation efficiency of $87 \%$ while these numbers for beams 2 and 3 are $7.5 \mathrm{dBi}$ and $75 \%$ respectively at $76.5 \mathrm{GHz}$.

To analyze the MIMO performance of antennas, it is common to calculate the envelope correlation coefficient between the ports. The two-by-two envelope correlation for the designed antenna by using the radiation pattern [15], [16] is calculated and shown in Fig. 5. It is shown that $\rho_{n m}$ is less than 0.03 for all ports. Those correlation coefficients that are not reported in the figure are the same as ones presented since the antenna structure is symmetric; for instance $\rho_{12}=\rho_{34}$ and $\rho_{13}=\rho_{24}$.

\section{CONCLUSION}

A balanced antipodal Vivaldi antenna in millimeter-wave band for self-driving cars is designed. The antenna provides pattern diversity, appropriate to be used in multiple-inputmultiple output (MIMO) channels. The view angle of the antenna is $90 \mathrm{deg}$ and by utilizing four of the designed antenna on four corners of a car, a total $360 \mathrm{deg}$ view will be achieved. The antenna performance is studied using full-wave packages and the simulation results show the proper behavior of the antenna. Although developed for self-driving cars, the proposed antenna design can find applications other than autonomous vehicles where MIMO multiplexing or diversity is required.

\section{REFERENCES}

[1] B. Brown, "The Social Life of Autonomous Cars," Computer, vol. 50, pp. 92-96, Feb. 2017.

[2] J. Khoury, R. Ramanathan, D. McCloskey, R. Smith, T. Campbell, "RadarMAC: Mitigating Radar Interference in Self-Driving Cars," $13^{\text {th }}$ Annual IEEE International Conference of Sensing, Communication, and Networking (SECON), Nov. 2016.

[3] Electronic Code of Federal Regulations. http://www.ecfr.gov, Title 47, Chapter 1, Subchapter A, Part 15, Subpart C, Section 15.253, Mar. 2015.

[4] M. Khalily, R. Tafazolli, T. A. Rahman, M. R. Kamarudin, 'Design of Phased Arrays of Series-Fed Patch Antennas with Reduced Number of the Controllers for 28-GHz mm-Wave Applications,' IEEE Antenna and Wireless Propagation Letters. vol .15, pp. 1305-1308, 2016.

[5] M. M. Molu, P. Xiao, M. Khalily, K. Cumanan, L. Zhang, R. Tafazolli, "Low-Complexity and Robust Hybrid Beamforming Design for MultiAntenna Communication Systems" IEEE Transactions on Wireless Communications, Volume: 17, Issue: 3, pp. 1445-1459, 2018.

[6] J. Wenger, M. Stotz, H. Barth, H. Neef, G. Wanielik, R. Schneider, "A Polarimetric $76 \mathrm{GHz}$ Radar-Sensor for Automotive Applications," $27^{\text {th }}$ European Microwave Conference, pp. 832-837, Sept. 1997.

[7] K. Yamaguchi, H. Nakajima, H. H. N. Pham, S. Hisatake, T. Nagatsuma, H. Uchida, M. Tojo, Y. Oikawa, K. Miyaji, "Electric-Field Visualization Technique for Evaluating Radiation from Automotive Millimeter-Wave Radar," IEEE MTT-S International Conference on Microwaves for Intelligent Mobility (ICMIM), pp. 151-154, Mar. 2017.

[8] D. Belgiovane, C. Chen, "Bicycles and Human Riders Backscattering at $77 \mathrm{GHz}$ for Automotive Radar," $10^{\text {th }}$ European Conference on Antennas and Propagation (EuCAP), Apr. 2016.

[9] A. Araghi, G. Dadashzadeh, "Oriented Design of an Antenna for MIMO Applications Using Theory of Characteristic Modes," IEEE Antennas and Wireless Propagation Letters, vol. 11, pp. 1040-1043, Aug. 2012.

[10] P. J. Gibson, "The Vivaldi Aerial," $9^{\text {th }}$ European Microwave Conference, pp. 101-105, Sep. 1979.

[11] J. D. S. Langley, P. S. Hall, P. Newham, "Balanced Antipodal Vivaldi Antenna for Wide Bandwidth Phased Arrays," IEE ProceedingsMicrowaves, Antennas and Propagation, vol. 143, pp. 97-102, Apr. 1996.

[12] Y. Zeng, C. Guo, X. Lin, X. Yang, "Vivaldi Antenna Design in Millimeter-Wave-Band with Ultra Wide Bandwidth and High Gain," IEEE MTT-S International Microwave Workshop Series on Advanced Materials and Processes for RF and THz Applications (IMWS-AMP), Jul. 2016.

[13] B. Tehrani, B. Cook, J. Cooper, M. Tentzeris, "Inkjet Printing of a Wideband, High Gain MM-Wave Vivaldi Antenna on a Flexible Organic Substrate," IEEE Antennas and Propagation Society International Symposium (APSURSI), Jul. 2014.

[14] G. Brzezina, R. E. Amaya, A. Petosa, L. Roy, "Broadband and Compact Vivaldi Arrays in LTCC for $60 \mathrm{GHz}$ Point-to-Point Networks," IEEE $15^{\text {th }}$ Annual Wireless and Microwave Technology Conference (WAMICON), Jun. 2014.

[15] S. Blanch, J. Romeu, I, Corbella, "Exact Representation of Antenna System Diversity Performance from Input Parameter Description," Electronic Letters, vol. 39, pp. 705-707, May 2003.

[16] R. G. Vaughan, J. B. Andersen, "Antenna Diversity in Mobile Communications," IEEE Transactions on Vehicular Technology, vol. 36, pp. 149-172, Nov. 1987. 